\title{
LINE PROFILES IN SUNSPOT UMBRAE AND PENUMBRAE BY ATOMIC BEAM SPECTROSCOPY
}

\author{
F. RODDIER
}

Faculté des Sciences de Nice, Laboratoire d'Astrophysique, Nice, France

We present profiles of the $\mathrm{Sr}$ resonance line obtained with an atomic beam spectrograph and the Kitt Peak main heliostat.

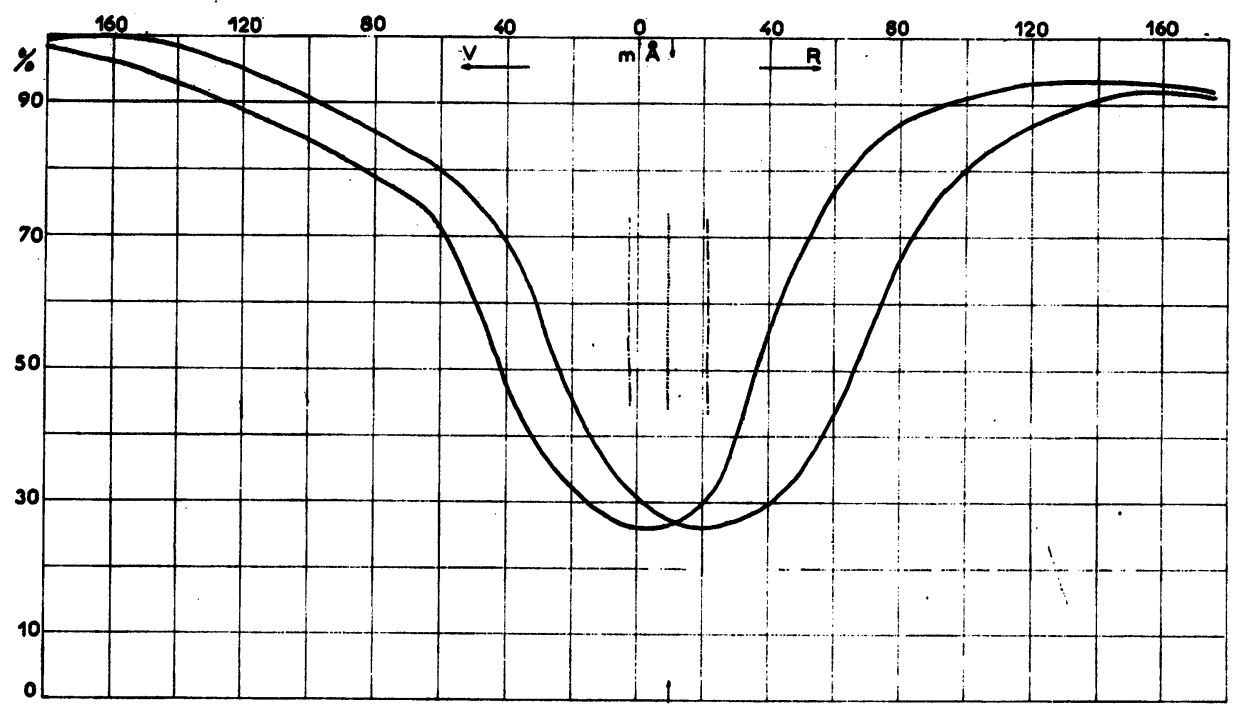

Fig. 1.

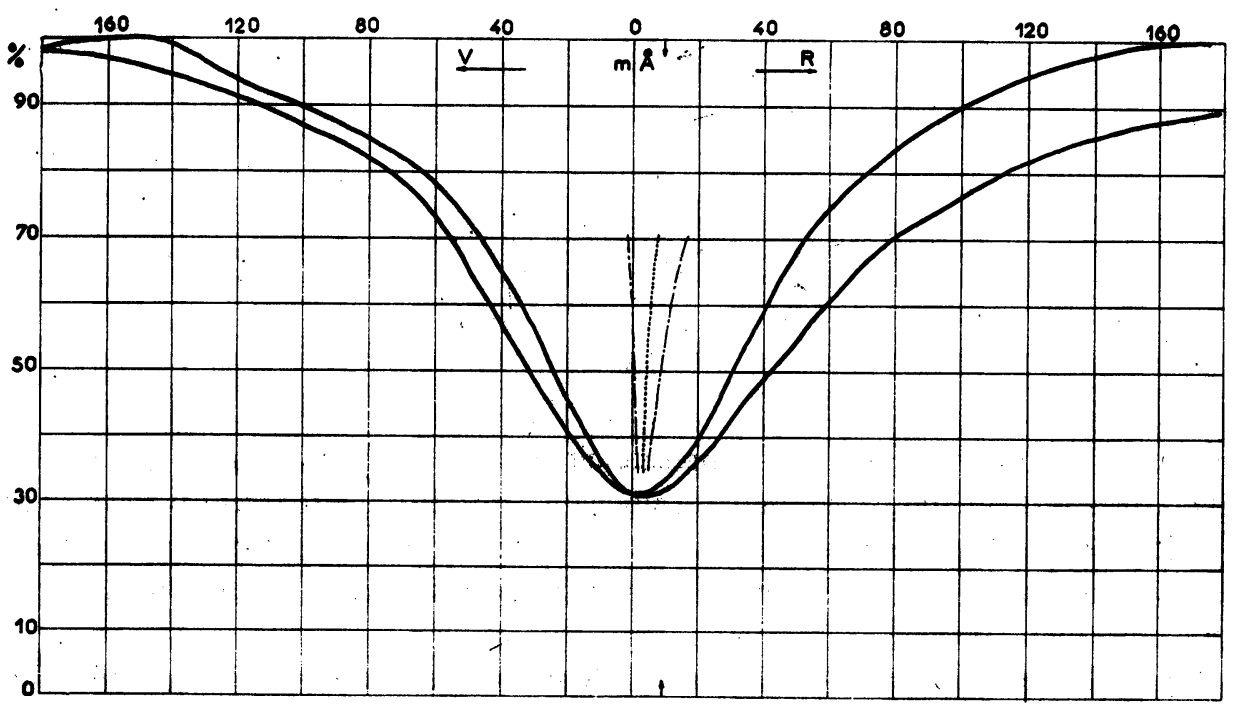

Fit. 2. 


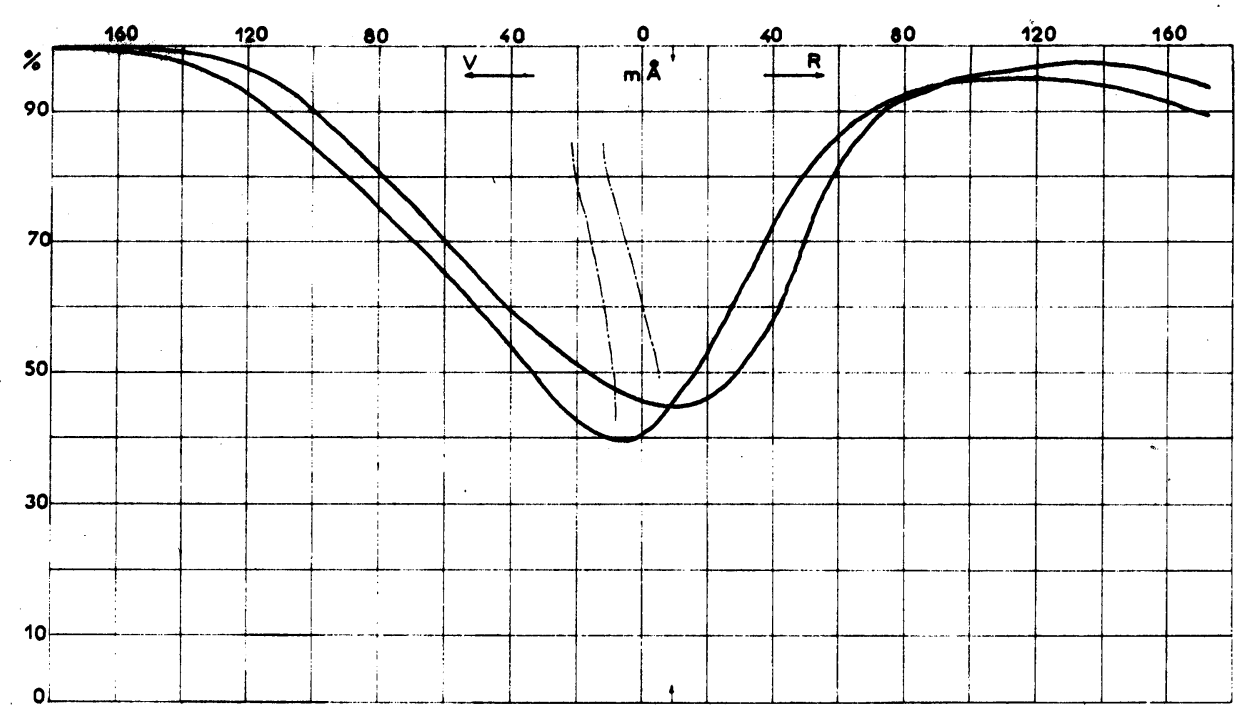

Fig. 3.

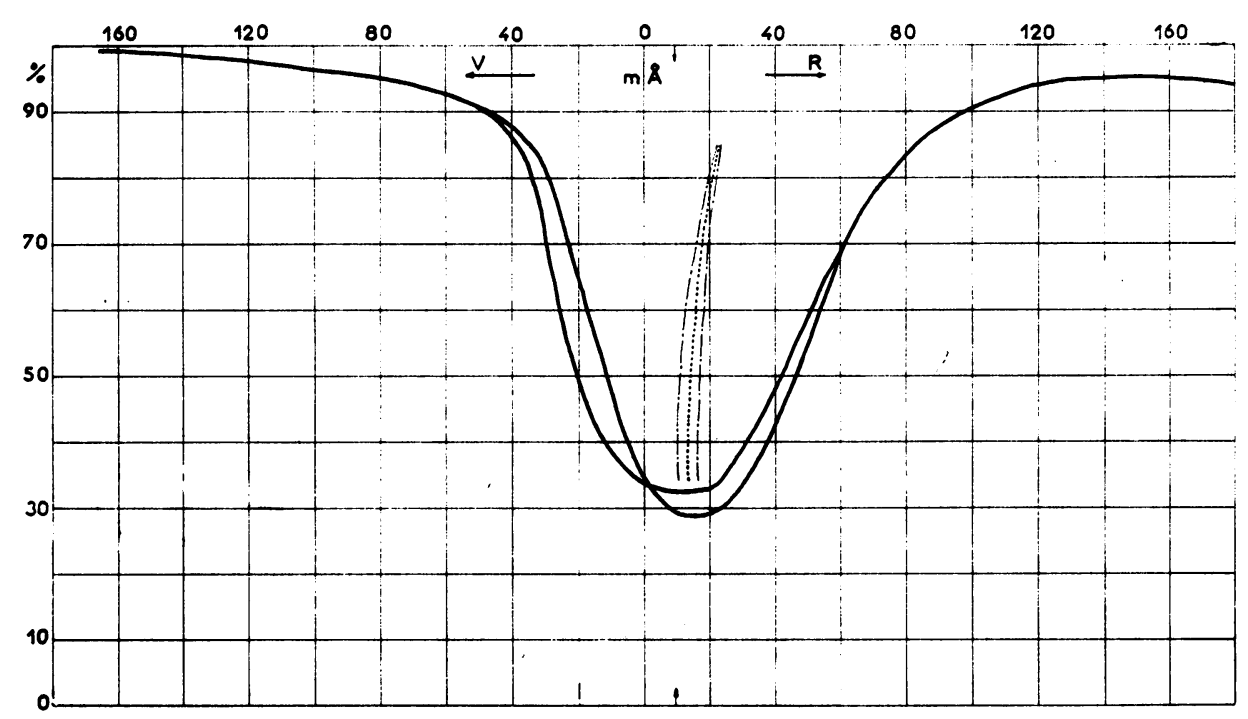

Fig. 4.

Figure 1 shows two profiles of opposite circular polarization in a sunspot umbra. The axis is vertical and split symmetrically with respect to the theoretical wavelength predicted by the Einstein theory of gravitation (arrow). This seems to show the absence of convective motion in the umbra. The splitting corresponds to a magnetic field of a little more than $1000 \mathrm{G}$.

Figure 2 shows the same profiles in a different sunspot umbra. We see a resulting violet shift decreasing toward the continuum while the splitting increases. This may 
show both magnetic field and convection with opposite vertical gradients, the magnetic field increasing with depth while the convection decreases.

Figure 3 shows these profiles in a sunspot penumbra between the umbra and the center of the solar disk. Both shift and assymmetry are due to the Evershed effect. Velocities are now increasing with depth. There is a systematic difference between the widths and central intensities of the two profiles which can be explained by a negative correlation between velocities and magnetic fields in the penumbral structures.

Figure 4 shows the same profiles in the penumbra between the umbra and the solar limb. We can see the same effects reversed.

\section{Discussion}

Wiehr: I want to mention that the increase of Evershed asymmetry towards the continuum does not come from an increase of the velocity with optical depth. Schröter showed in 1965 that the asymmetry comes from a satellite which is superimposed on the main line. 